\title{
Graphene based Fiber Optic Surface Plasmon Resonance for Bio-chemical sensor Applications
}

\author{
Jang Ah Kim ${ }^{1}$, Taehyun Hwang ${ }^{3}$, Rashid Amin ${ }^{1}$, Sungha Park ${ }^{2}$, Atul Kulkarni ${ }^{2,3 *}$ and Taesung Kim ${ }^{1,3 * *}$ \\ ${ }^{1}$ SKKU Advanced Institute of Nano Technology (SAINT), Sungkyunkwan University, \\ Suwon 440-746, Korea \\ ${ }^{2}$ Department of Physics, Sungkyunkwan University, Suwon 440-746, Korea \\ ${ }^{3}$ Department of Mechanical Engineering, Sungkyunkwan University, Suwon 440-746, Korea \\ *atulnano@skku.edu and **tkim@skku.edu
}

\begin{abstract}
:
In this study, a surface plasmon resonance (SPR) based fiber optic sensor coated with graphene is described. Graphene synthesized by chemical vapor deposition (CVD) is transferred on sensing area of an optical fiber. The sensor principle is based on change in SPR according to the different dielectric constants of analytes. This sensor is evaluated with biotinylated DNA lattice and protein Streptavidin. To the best of our knowledge, this is the first ever attempt to incorporate graphene as the replacement of conventional metal films. The preliminary result is encouraging results towards the analytes with excellent sensitivity. We believe that the use of graphene as a metallic film in SPR sensor will lead new path in the field of biochemical sensing technology.
\end{abstract}

Key words: surface plasmon resonance (SPR), Graphene, optical fiber, biotinylated DNA lattice, Streptavidin

\section{Introduction}

The optical fibers have facilitated more numerous benefits such as potential for the development of miniaturized, compact, rugged sensing elements, and multiple sensing using multi-fibers on one chip in biosensors [1]. Surface plasmon resonance (SPR) sensors are the most advantageous sensors for bio-chemical materials because pre-processing, such as fluorescence labeling, is not required as well as real-time analysis is possible [2]. For the conventional SPR sensors metallic films such as gold and silver films have been utilized due to their dielectric functions leading sensitivity and accuracy is appropriate. However, the poor adsorption of biomaterials, such as proteins, makes the limitation of the performance of gold film. There are several methods to improve the adsorption such as using nanoparticles and nanoholes, metallic nanoslits, and biomolecular recognition elements (BRE) [3].

Meanwhile, as the most spotlighted material due to its remarkable mechanical and electrical characteristics and advantages such as flexibility, transparency, and surface-to-volume ratio (SVR), graphene can be adapted to various fields with its well established and simple production process [4]. Especially, according to the high SVR, it is possible to enhance an interaction of graphene with other materials. Therefore, use of graphene in various sensor applications can be a great groundbreaking investigation. In this study, use of graphene as a metal film for SPR sensor is evaluated.

\section{Experimental}

First of all, graphene film is synthesized by thermal chemical vapor deposition (CVD) method with the reaction gases, methane and hydrogen, on the copper foil as a catalyst substrate. Meanwhile, polymer optical fiber (POF) is used as the SPR sensor platform. The synthesized graphene film is transferred on the sensing area $(\sim 10 \mathrm{~mm})$ of the POF. The sensor probe comprising graphene coated sensing region is shown in Fig. 1 (a).

Figure 1 (b) describes the schematic of experimental setup employed in this study. As light source, blue light which has $460 \mathrm{~nm}$ of wavelength is used. To produce the TM mode light input, the optics, such as, beam splitter, convex lens, and linear polarizer are utilized. The output light is collected by spectrometer calculating the intensity of light toward the wavelength and PC displays the signals. 


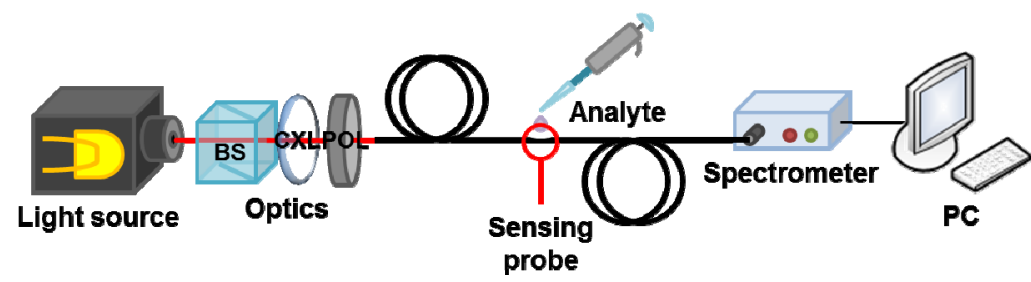

(a)

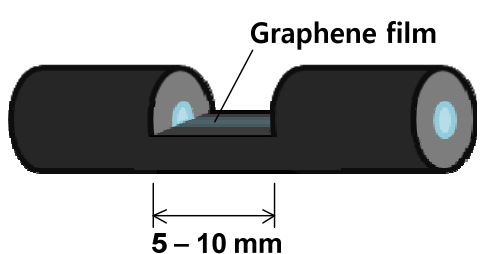

(b)

Fig. 1. Schematic of (a) experimental setup consisting of light source, required optics, spectrometer and PC for analysis of the output sensor signal. (b) The configuration of optical fiber sensor probe with graphene film.

The biotinylated DX (DX-Biotin) lattice is designed based on the structure of immobile crossover branched junctions, and the DNA base sequence is designed. DNA oligonucleotides were obtained from Integrated DNA Technologies (Coralville, IA, USA). The final concentration of the DNA structure used was 400nM. Streptavidin (SA) was purchased from Rockland Inc. (PA, USA). A 400 nM solution of streptavidin was prepared in deionized water.

\section{Results and Discussion}

Figure 2 shows the preliminary results with the difference between bare fiber and graphene coated fiber. We observed different depending on two types of fibers with the used bioanalytes. For the bare fiber, there are two peaks, upper and lower directed, and the intensity differences between two peaks are little. Therefore, we can know that the signals of the bare fiber come from the only evanescent field. On the other hand, the signals of the graphene coated fiber have one downward dip due to the resonance , further they are extremely larger than the bare fiber's ones. From these observations, we can conclude that surface plasmon resonance takes place in the deposited metallic graphene film on the optical fiber. In addition, there is shift in the peak wavelength depending upon the biochemical analytes used, which confirms the SPR phenomena. Further evaluation with other biochemical analytes is in process. Hence the proposed graphene based SPR sensor can be utilized as a sensitive SPR sensor for biochemical sensor applications.

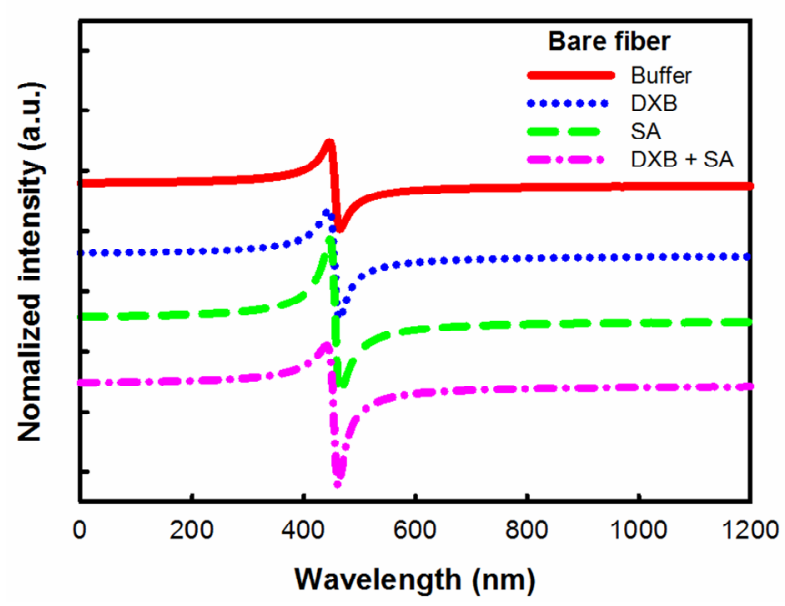

(a)

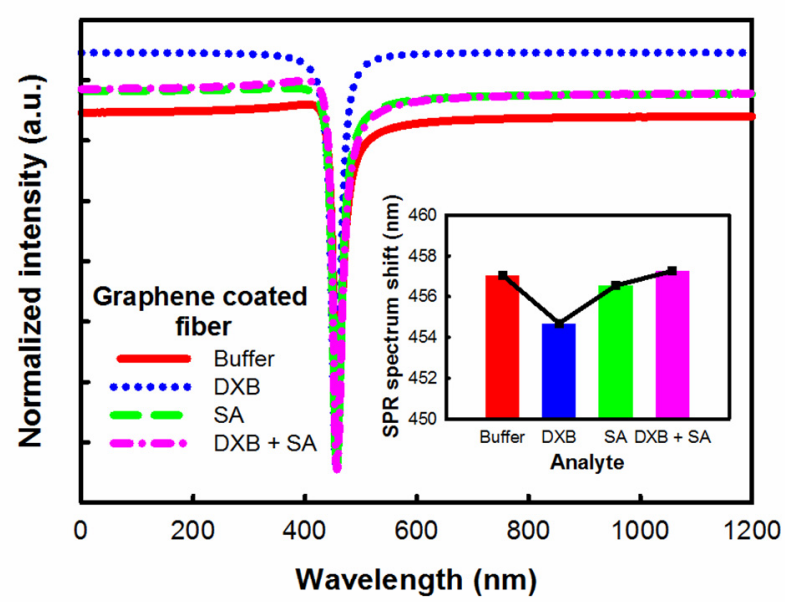

(b)

Fig. 2. SPR response for (a) without coated graphene fiber and (b) graphene coated fiber showing the SPR spectrum shift (inset image).

\section{Acknowledgements}

This work was partly supported by the GRRC program of Gyeonggi province [S-2011-1039007-1, Development of integrated sensor module for measurement of TVOCs and PM2.5 from vehicle emission] and Basic Science Research Program through the National Research Foundation of Korea(NRF) funded by the Ministry of Education, Science and Technology (2009-0083540) 


\section{References}

[1] J. Dostálek, J. Čtyroký, J. Homola, E. Brynda, M. Skalský, P. Nekvindová, J. Špirková, J. Škvor and J. Schröfel, Surface plasmon resonance biosensor based on integrated optical waveguide, Sensors and Actuators B 76, 8-12 (2001); doi:10.1016/s0925-4005(01)00559-7

[2] J. Homola, Present and future of surface plasmon resonance biosensors, Analytical Bioanalytical Chemistry 377, 528-539 (2003); doi: 10.1007/s00216-003-2101-0

[3] J. Zhao, X. Zhang, C. R. Yonzon, A. J. Haes, and R. P. Van Duyne, Localized surface plasmon resonance biosensors, Nanomedicine (London, England) 1, 219-228 (2006); doi: 10.2217/17435889.1.2.219

[4] A. K. Geim, K.S. Novoselov, The rise of graphene, Nature Materials 6, 183-191 (2007); doi: 10.1038/nmat1849 